\title{
Prevalence and predictors of burnout among obstetrics and gynecology residents in Canada
}

\author{
Na'ama O. Al-Ma'mari ${ }^{1} \cdot$ Ashley I. Naimi ${ }^{1} \cdot$ Togas Tulandi $^{1}$
}

Received: 1 November 2015 / Accepted: 4 May 2016/Published online: 20 May 2016

(C) Springer-Verlag Berlin Heidelberg 2016

\begin{abstract}
The aim of this study is to evaluate the prevalence of burnout syndrome among obstetrics and gynecology residents, as well as the relationship between several demographic and work-related characteristics and the prevalence of burnout. We surveyed 143 residents in obstetrics and gynecology programs across eight provinces in Canada using an electronic survey questionnaire and the Maslach Burnout Inventory Human services survey. The prevalence of high emotional exhaustion was $12.6 \%$ higher among younger than older residents and $12.4 \%$ higher among female compared to male residents. The prevalence of high depersonalization was $13.0 \%$ higher among male versus female residents. The prevalence of high emotional exhaustion among residents who reported sufficient supervision during the night shift was significantly lower than among those who reported insufficient supervision (95\% CI -49.7, -8.6). Additionally, we found that adequate access to food during the night shift was associated with a lower prevalence of any burnout $(95 \%$ CI $-31.8,-0.2)$. Similar associations were observed for high emotional exhaustion and high depersonalization. We also found a $22.1 \%(95 \% \mathrm{CI}-60.0,15.7)$ lower prevalence of emotional exhaustion among residents who work $60 \mathrm{~h} /$ week
\end{abstract}

Condensation: Decreased levels of burnout in residents with sufficient staff supervision, adequate access to food during the night shift, and fewer working hours.

Electronic supplementary material The online version of this article (doi:10.1007/s10397-016-0955-3) contains supplementary material, which is available to authorized users.

Togas Tulandi togas.tulandi@mcgill.ca

1 Department of Obstetrics and Gynecology, McGill University, 1001 Boulevard Decarie, Montreal, QC H4A 3J1, Canada or less. Our results suggest decreased levels of burnout in resident physicians who reported sufficient staff supervision, adequate access to food during the night shift, and fewer working hours. Future studies should be directed to examine the effectiveness of different strategies to improve the residency training in obstetrics and gynecology.

Keywords Burnout · Residents · Obstetrics and gynecology

The physical and emotional stress of residency training can have a lasting negative impact on resident well-being and patient care [1]. These stresses can lead to emotional and psychological burnout, decreased physician productivity [2], impaired job performance, and poor social relationships [3]. The stress of residency can result in burnout syndrome, a pathological emotional depletion and maladaptive detachment syndrome secondary to prolonged stress [4]. There are three aspects of this syndrome, emotional exhaustion (characterized by energy depletion), depersonalization (wherein the individual detaches from the job), and low personal accomplishment and feeling of inefficacy $[5,6]$.

Stress is common among health care professionals. Compared to the general population, physicians have more consequences of burnout including divorce and substance abuse [7]. In addition, residents affected by burnout are more likely to self-report suboptimal patient care practices [8].

Improving resident physical and emotional well-being is challenging. Different approaches have been taken to optimize resident's well-being without affecting patient care, including a limitation of resident duty hours, known in the USA as the Lippy Zion Law. The death of Lippy Zion has been attributed to resident fatigue, overwork, and lack of experience [9]. In July 2003, the Accreditation Council for Graduate Medical Education (ACGME) in the USA mandated that the number 
of resident duty hours be limited to 80 or less per week. In 2011, the ACGME restricted the residents duty hours up to $16 \mathrm{~h}$ for postgraduate year 1 (PGY-1) [10].

There have been no Canadian guidelines on duty hour restrictions [11]. However, each province has its own regulations. For example, in Quebec, recent regulations have limited the number of residents' duty hours to no more than $16 \mathrm{~h}$ in an effort to reduce excessive burdens for residents and optimize patient care [12]. The Royal College of Physicians and Surgeons of Canada National Steering Committee recommended institutional strategies such as wellness programs to extenuate fatigue and improve physical, emotional, and mental well-being [10]. However, the evidence base supporting these recommendations is limited.

Few studies have examined the prevalence and predictors of burnout among medical residents in Canada: two studies among medical residents in Alberta and Ontario [13, 14]. However, no research has focused on obstetrics and gynecology residents nor examined whether the prevalence of burnout varies across Canadian provinces. Moreover, research to date has been limited to a small range of predictors of burnout.

The purpose of our study was to explore the prevalence of burnout syndrome among 143 Obstetrics and Gynecology residents in eight Canadian provinces using a validated psychometric questionnaire [15]. We also evaluated the relationship between several demographic and work-related characteristics with an increased prevalence of burnout. Detecting the prevalence of burnout syndrome and assessing contributing factors can be used to improve residents' well-being and subsequently to enhance patient care and ensure safe work environment.

\section{Methods}

We surveyed 143 residents in Obstetrics and Gynecology residency programs across eight provinces in Canada using an electronic survey questionnaire. The residency office in each program was contacted to distribute the web-based questionnaire to their residents. The psychometric questionnaire consisted of three parts. Part one included social and demographic data (age, gender, marital status, and residency level), part two included work-related predisposing factors (number of working hours, call schedule, night float system, lack of sleep, relationships in work environment, and lack of appreciation of your work), and part three included the Maslach burnout inventory (MBI) questionnaires [15]. The Research Ethics Committee of the Jewish General Hospital approved the study (Protocol 13-120).

The MBI has 22 questions to measure the level of burnout in three domains: emotional exhaustion, depersonalization, and personal accomplishment. High/low scores in personal accomplishment subscales and low/high scores of both emotional exhaustion and depersonalization indicate low/high levels of burnout. For factors contributing to burnout, the answer to a question could be "1. Does not apply, 2. Minimally applies, 3. Neutral, 4. Moderately applies, or 5. Strongly applies." For Maslach burnout inventory, the score could be "0 Never, 1 Once a year or less, 2 Once a month or less, 3 Sometimes in a month, 4 Once a week, 5 Sometimes in a week, or 6 Everyday." Average scores in all subscales reflect an average degree of burnout.

Of 16 residency programs, 12 programs with a total 373 residents responded. The proportion of male residents among all programs was $16 \%$. One hundred and forty-three completed the survey of all responses, and 26 (18\%) had missing or incomplete information. We used multiple imputation by chained equations [16] to account for these missing data, with 20 imputations. Measures of depersonalization and emotional exhaustion were dichotomized into high versus medium/low according to predetermined criteria [6]. The measure of personal accomplishment was dichotomized into low versus medium/high according to similar criteria.

We also defined a fourth binary variable equal to 1 if any of these domains were lower (personal accomplishment) or higher (emotional exhaustion, depersonalization) than their prespecified cut-points according to predetermined criteria. This allowed us to assess whether certain characteristics are associated with any features related to burnout. We used binomial regression to estimate associations between several characteristics (demographic and work related), and the prevalence of high emotional exhaustion or depersonalization, or low personal accomplishment. "Any burnout" was defined as the presence of burnout related to emotional exhaustion or depersonalization or personal accomplishment. In contrast to a previous definition in which an individual had to score high enough on all three scales, a person who scored high or low enough on any of these scales was considered "burned out." Comparison of groups was done using Wald test. Analyses were adjusted for age, sex, and marital status. All analyses were conducted in $\mathrm{R}$ version 3.2.1 [17].

\section{Results}

Over one third of residents responded to the survey. This was a good response rate for an external survey. Overall, $13 \%$ of the respondents were males, $37 \%$ were married, and $40 \%$ were in year 1 or 2 of their residency training. Additionally, 64, 51, and $25 \%$ of them were found to have high levels of emotional exhaustion, high levels of depersonalization, and a low sense of personal accomplishment, respectively. Furthermore, 16, 32 , and $31 \%$ of the respondents were residents in Alberta, Ontario, and Quebec, respectively.

Table 1 shows the proportion of obstetrics and gynecology residents with high levels of emotional exhaustion, high levels of depersonalization, and a low sense of personal 
Table 1 Proportion of obstetrics and gynecology residents with high levels of emotional exhaustion, high levels of depersonalization, and a low sense of personal accomplishment from eight Canadian provinces $(N=143$ residents)

\begin{tabular}{|c|c|c|c|c|}
\hline & $\begin{array}{l}\text { High emotional } \\
\text { exhaustion }(\%)\end{array}$ & $\begin{array}{l}\text { High depersonalization } \\
(\%)\end{array}$ & $\begin{array}{l}\text { Low personal } \\
\text { accomplishment (\%) }\end{array}$ & $\begin{array}{l}\text { Any burnout } \\
(\%)\end{array}$ \\
\hline Overall & 64.1 & 50.7 & 24.5 & 73.7 \\
\hline \multicolumn{5}{|l|}{ Age } \\
\hline$\leq 30$ years & 67.8 & 52.7 & 27.6 & 76.0 \\
\hline$>30$ years & 55.1 & 45.8 & 17.1 & 67.9 \\
\hline \multicolumn{5}{|l|}{ Gender } \\
\hline Male & 53.2 & 62.0 & 40.1 & 72.2 \\
\hline Female & 65.8 & 49.0 & 22.2 & 73.9 \\
\hline \multicolumn{5}{|l|}{ Married } \\
\hline Yes & 64.4 & 50.1 & 22.6 & 71.0 \\
\hline No & 63.9 & 51.0 & 25.6 & 75.2 \\
\hline \multicolumn{5}{|l|}{ Residency year } \\
\hline $\mathrm{R} 2$ or less & 67.7 & 50.3 & 30.7 & 76.3 \\
\hline $\mathrm{R} 3$ or more & 61.7 & 51.0 & 20.4 & 71.9 \\
\hline \multicolumn{5}{|l|}{ Province } \\
\hline Alberta & 72.4 & 52.5 & 18.5 & 75.3 \\
\hline Ontario & 66.1 & 44.7 & 22.1 & 71.2 \\
\hline Quebec & 60.9 & 53.4 & 30.0 & 72.6 \\
\hline Other & 59.6 & 54.3 & 24.5 & 75.6 \\
\hline
\end{tabular}

accomplishment stratified by several demographic covariates. The prevalence of high emotional exhaustion was $12.6 \%$ higher among younger (age 30 years or less) compared to older residents $(p=0.19), 12.4 \%$ higher among female compared to male residents $(p=0.38)$, and not different between married versus unmarried residents (prevalence difference $=0.5 \%, p=0.95$ ). Alternatively, the prevalence of high depersonalization was $13.0 \%$ higher among male versus female residents $(p=0.33)$. No differences in the prevalence of high depersonalization were observed between older and younger residents, or between married versus unmarried residents. Finally, the prevalence of a low sense of personal accomplishment was $10.6 \%$ lower among younger compared to older residents $(p=0.24)$ and $17.8 \%$ higher among males $(p=0.20)$. These differences, however, while substantively relevant, were not statistically significant at the $\alpha=0.05$ level.

Table 2 shows prevalence differences associated with several characteristics. We found that the prevalence of high emotional exhaustion among residents who reported sufficient supervision during the night shift was $29.2 \%$ (95\% CI -49.7, -8.6) lower, compared to residents who reported insufficient supervision. Similar differences were observed for the prevalence of high depersonalization, low personal accomplishment, or any burnout (burnout not specifically related to emotional exhaustion, depersonalization, or personal accomplishment). Additionally, we found that adequate access to food during the night shift was associated with a $16 \%(95 \% \mathrm{CI}$ $-31.8,-0.2)$ lower prevalence of any burnout. Similar associations were observed for high emotional exhaustion and high depersonalization. We also found a $22.1 \%(95 \%$ CI -60.0, 15.7) lower prevalence of emotional exhaustion among residents that work $60 \mathrm{~h}$ /week or less. In addition, this lower prevalence occurred mostly among the third to fifth year residents (R3-R5), relative to those in the first and second year of residency (R1-R2), as evidenced by a large but statistically nonsignificant interaction term. Night float was associated with an $11.4 \%(95 \% \mathrm{CI}-5.7,28.5)$ higher prevalence of emotional exhaustion, and being able to sleep during night shift was associated with a $13.2 \%(95 \%-32.9,6.5)$ lower prevalence of emotional exhaustion.

\section{Discussion}

Burnout among resident physicians may have important consequences on patient care [1]. In our study, we found that the prevalence of high emotional exhaustion among residents who reported sufficient supervision during the night shift was nearly $30 \%$ lower than residents who reported insufficient supervision. Similar differences were observed for the prevalence of high depersonalization, low personal accomplishment, or any burnout. This finding coincides with a previous study of eight Spanish University Hospitals, where lack of staff supervision was a significant predictor of burnout [4]. Availability of the attending staff may provide an important sense of security and bolster confidence, especially in situations where unexpected emergencies can be relatively common. 
Table 2 Prevalence differences of high emotional exhaustion, high depersonalization, low personal accomplishment, and any burnout among obstetrics and gynecology residents from eight Canadian Provinces $(N=143$ residents $)$

\begin{tabular}{lllll}
\hline & $\begin{array}{l}\text { High emotional exhaustion } \\
\text { \% Diff }(95 \% \text { CI })\end{array}$ & $\begin{array}{l}\text { High depersonalization } \\
\text { \% Diff }(95 \% \text { CI) }\end{array}$ & $\begin{array}{l}\text { Low personal accomplishment } \\
\% \text { Diff }(95 \% \text { CI })\end{array}$ & $\begin{array}{l}\text { Any burnout } \\
\% \text { Diff }(95 \% \text { CI) }\end{array}$ \\
\hline Local vs. international & $-12.2(-58.2,33.7)$ & $-20.3(-69.2,28.6)$ & $-13.7(-57.9,30.5)$ & $-27.9(-93.7,37.8)$ \\
Work <60 h/week vs. other & $-22.1(-60.0,15.7)$ & $0.3(-34.9,34.9)$ & $11.7(-24.6,48.0)$ & $-3.8(-30.8,23.2)$ \\
24-h call vs. none & $-4.7(-24.0,14.6)$ & $-0.7(-20.7,19.2)$ & $-3.7(-20.5,13.1)$ & $-9.4(-26.3,7.5)$ \\
Night float vs. none & $11.4(-5.7,28.5)$ & $8.4(-11.4,28.2)$ & $7.7(-9.9,25.4)$ & $15.4(-0.6,31.4)$ \\
Any sleep vs. none & $-13.2(-32.9,6.5)$ & $-4.9(-25.7,16.0)$ & $1.0(-20.0,21.9)$ & $-7.0(-23.4,9.3)$ \\
No post-call duties vs. any & $-5.4(-39.2,28.5)$ & $5.2(-28.9,39.2)$ & $-21.4(-59.2,16.3)$ & $-7.1(-31.0,16.9)$ \\
Supervised vs. unsupervised night shift & $-29.2(-49.7,-8.6)$ & $-18.8(-41.7,4.1)$ & $-21.7(-45.4,2.0)$ & $-28.6(-47.0,-10.2)$ \\
Adequate vs. inadequate access to food & $-12.3(-30.8,6.2)$ & $-15.0(-32.7,2.7)$ & $-4.7(-22.0,12.7)$ & $-16.0(-31.8,-0.2)$ \\
Adequate vs. inadequate access to rooms & $-4.1(-24.2,16.0)$ & $-8.5(-28.6,11.7)$ & $4.5(-16.3,25.3)$ & $-10.5(-27.8,6.9)$ \\
\hline
\end{tabular}

When the $95 \%$ CI does not include 1.0, the difference is significant at 0.05 level

Access to food during the night shift is associated with $16 \%$ lower prevalence of any levels of burnout. In many teaching hospitals, availability of food outlets (cafeterias, cafes, etc.) is reduced after normal operating hours. To our knowledge, no previous studies have demonstrated a relationship between adequate access to food during the night shift and resident burnout. This finding suggests the relatively simple strategy of ensuring adequate provision of nutritional resources after normal hospital operating hours may alleviate some of the excess burdens of residency training. Yet, instead of access to food only, other factors such as the provision of a break during the night shift or better hospital accommodation might play a role.

Further, we found $22 \%$ lower prevalence of emotional exhaustion or any burnout among residents who work $60 \mathrm{~h} /$ week or less. In a previous study, an increasing level of responsibility that comes with higher residency level, rather than long working hours, was associated with higher level of burnout [18]. Yet, it is also possible that long working hours interact with increasing levels of responsibility.

The findings of our study suggest that certain relatively simple strategies should be further explored as avenues to optimize residency burnout. These include increasing attendant physician supervision during the nightshift, increasing availability of appropriate meals during the nightshift, and possibly altering work schedules such that residents work no more than $60 \mathrm{~h} /$ week. These strategies should be more rigorously assessed in future studies, preferably using some form of randomized intervention trial (such as stepped wedge design) [19].

Our results should be interpreted in light of certain limitations. As with most studies [20], ours was underpowered to detect a significant interaction between the number of work hours and residency level. However, the magnitude and direction of the interaction we estimated lend credence to this notion. In particular, we used an online volunteer-based survey questionnaire, which made it difficult to quantify the response rate and may have induced selection bias. However, many of our findings coincide with previous research in other settings. Additionally, we are the first to assess the status of residents in several obstetrics and gynecology training programs across Canada, and our results shed light on actionable strategies to optimize training. Future studies should be directed to examine the effectiveness of different strategies to improve the residency training in obstetrics and gynecology.

Acknowledgments We would like to thank the program directors of Obstetrics and Gynecology, coordinators, and participating residents for their cooperation in this study. Special acknowledgement is given to Alessandra Celani, the residency program coordinator at McGill University for her assistance in distributing our survey to all Canadian residency programs.

\section{Compliance with ethical standards}

Conflict of interest Drs. Al-Mamari and Naimi have no conflict of interest

Dr. Tulandi is an advisor for Actavis Inc.

\section{References}

1. Lund KJ, Alvero R, Teal SB (2004) Resident job satisfaction: will 80 hours make a difference? Am J Obstet Gynecol 191:1805-1810

2. Dewa CS, Loong D, Bonato S, Thanh NX, Jacobs P (2014) How does burnout affect physician productivity? A systematic literature review. BMC Health Serv Res 14:325

3. Becker JL, Milad MP, Klock SC (2006) Burnout, depression, and career satisfaction: cross-sectional study of obstetrics and gynecology residents. Am J Obstet Gynecol 195:1444-1449

4. Castelo-Branco C, Figueras F, Eixarch E, Quereda F, Cancelo MJ, Gonzalez S et al (2007) Stress symptoms and burnout in obstetrics and gynaecology residents. BJOG 114:94-98

5. Thomas NK (2004) Resident burnout. JAMA 292:2880-2889 
6. Christina M, Jackson SE, Leiter MP (1996) Maslach Burnout Inventory Manual. Third ed. Consulting Psychologists Press, Mind Garden

7. Afzal KI, Khan FM, Mulla Z, Akins R, Ledger E, Giordano FL (2010) Primary language and cultural background as factors in resident burnout in medical specialties: a study in a bilingual US city. South Med J 103:607-615

8. Shanafelt TD, Bradley KA, Wipf JE, Back AL (2002) Burnout and self-reported patient care in an internal medicine residency program. Ann Int Med 136:358-367

9. Brensilver JM, Smith L, Lyttle CS (1998) Impact of the Libby Zion case on graduate medical education in internal medicine. Mount Sinai J Med 65:296-300

10. Ahmed N, Devitt KS, Keshet I, Spicer J, Imrie K, Feldman L et al (2014) A systematic review of the effects of resident duty hour restrictions in surgery: impact on resident wellness, training, and patient outcomes. Annals Surg 259:1041-1053

11. Masterson MF, Shrichand P, Maniate JM (2014) Resident duty hours in Canada: a survey and national statement. BMC Med Educ 14(1):S9

12. Fédération des médecins résidents du Québec, collective agreement (2010-2015) Call schedule and Duty. Article 12

13. Cohen JS, Patten S (2005) Well-being in residency training: a survey examining resident physician satisfaction both within and outside of residency training and mental health in Alberta. BMC Med Educ 5:21

14. Legassie J, Zibrowski EM, Goldszmidt MA (2008) Measuring resident well-being: impostorism and burnout syndrome in residency. $\mathrm{J}$ Gen Int Med 23:1090-1094

15. Kleijweg JH, Verbraak MJ, Van Dijk MK (2013) The clinical utility of the Maslach Burnout Inventory in a clinical population. Psychol Asses 25:435-441

16. White RP, Royston AMW (2011) Multiple imputation using chained equations: issues and guidance for practice. . Statistics in Medicine 377-99.

17. R core team. R: a language and environment for statistical computing (2014). R Foundation for Statistical computing, Vienna, Austria. Available from: http://www.R-project.org/. Accessed May $2,2015$.

18. Garza JA, Schneider KM, Promecene P, Monga M (2004) Burnout in residency: a statewide study. South Med J 97:1171-1173

19. Hussey MA, Hughes JP (2007) Design and analysis of stepped wedge cluster randomization trials. Contemp Clin Trials 28:182191

20. Marshall SW (2007) power for test of interaction: effect of raising the type I error rate. Epidemiol Perspect Innov 